\title{
Contingent Composition as Identity
}

\section{Abstract}

When the Necessity of Identity ( $\mathrm{NI})$ is combined with Composition as Identity (CAI), the contingency of composition (CC) is at risk. In the extant literature, either $\mathrm{NI}$ is seen as the basis for a refutation of $\mathrm{CAl}$ or $\mathrm{CAl}$ is associated with a theory of modality, such that: either $\mathrm{NI}$ is renounced (if counterpart theory is adopted); or CC is renounced (if the theory of modal parts is adopted). In this paper, we investigate the prospects of a new variety of $\mathrm{CAl}$, which aims to preserve both $\mathrm{NI}$ and $\mathrm{CC}$. This new variety of CAI (CCAI, Contingent Composition as identity) is the quite natural product of the attempt to make sense of CAI on the background of a broadly Kripkean view of modality, such that one and the same entity is allowed to exist at more than one possible world. CCAI introduces a worldrelative kind of identity, which is different from standard identity, and claims that composition is this kind of world-relative identity. CCAI manages to preserve $\mathrm{NI}$ and CC. We compare CCAI with Gibbard's and Gallois' doctrines of contingent identity and we show that CCAI can be sensibly interpreted as a form of Weak CAI, that is of the thesis that composition is not standard identity, yet is significantly similar to it.

\section{§ 1. Introduction}

A powerful argument proves that every instance of identity holds necessarily. On the other hand, many instances of composition, and in particular those involving concrete entities, plainly seem to hold contingently. In the middle sits Composition as Identity (CAI), rather uncomfortably: CAI is indeed the claim that every instance of composition is an instance of identity.

Here is an instance of the powerful argument which proves identity to be necessary. Everything is identical to itself. This claim does not depend on any peculiarity of the way in which things contingently are. Thus, it holds necessarily that everything is identical to itself. Take for example Trump. It also holds for him: Trump is necessarily identical to Trump. Thus, Trump has the property of being necessarily identical to Trump. Moreover, Trump is identical to Donald: Trump and Donald are one and the same individual. As a consequence, it does not make sense to think that there is a property or a relation such that Trump instantiates it and Donald lacks it. Thus, Donald shares with Trump also the property of being necessarily identical to Trump.

Nothing in this reasoning depends on any peculiarity of Trump/Donald. Thus, not only the identity of Trump and Donald, but also any other instance of identity holds necessarily. This argument has convinced the majority of philosophers of the Necessity of Identity (NI).

Let us now see why many instances of composition are plainly contingent. Consider the relation between Trump and the molecules composing Trump. Many of the molecules in the external layer of Trump's skin might have failed to be part of Trump. In this scenario Trump would not be composed by some entities which actually compose him. Many other events in the life of Trump might have gone differently: for example, he might have undergone an additional hair transplant, who would have caused many other molecules to become his parts. In this scenario, Trump would be composed by entities which do not actually compose him. Analogous examples might concern a cat, a chair, an island or any other concrete entity: their composition seems to be contingent as well. These reasonings support the Contingency of Composition (CC), that is the claim that many instances of composition are contingent.

According to CAI, composition is (or is significantly analogous to) identity. This quickly leads (or should lead) to ask:

How can composition be identity or significantly analogous to identity, if composition and identity so sharply differ in their modal status? 
It might seem that $\mathrm{NI}, \mathrm{CC}$ and $\mathrm{CAl}$ are an inconsistent set of thesis, and that, as a consequence, at least one of them should go:

Necessity of Identity (NI). Every instance of identity holds necessarily.

Contingency of Composition (CC). Many instances of composition hold contingently.

Composition as Identity (CAI). Composition is (or is significantly analogous to) identity.

The purpose of this paper is to discuss a new variety of CAI which avoids this inconsistency and is compatible both with $\mathrm{NI}$ and with CC.

By contrast, the extant literature on CAI, when it deals with the relation between CAI and $\mathrm{NI}$ at all, accepts that $\mathrm{NI}$, the contingency of composition and CAl are indeed an inconsistent of theses and goes in one of the following directions:

a) either CAI is rejected (Merricks 1999);

b) or $\mathrm{NI}$ is rejected, by endorsing a view of modality (counterpart theory) in which $\mathrm{NI}$ can fail (Bøhn 2009; Borghini 2005);

c) or CC is rejected, by endorsing a view of modality (theory of modal parts) that makes the resulting failure of CC palatable (Wallace 2014).

We will label the new variety of CAI we are going to discuss Contingent Composition as Identity (CCAI). CCAI claims that composition is a contingent variety of identity. CCAI emerges quite naturally when we assume as a background, in dealing with the modal notions at stake, a broadly Kripkean view of modality, such that an entity is allowed to exist at more than one possible world and inter-world identity is admitted. Given this background, composition - as any other standard property and relation - is relativized to worlds. In order to make sense of CAI while preserving this relativization, a relativized, contingent variety of identity is introduced. This relativized, contingent variety of identity does not replace standard, absolute identity, but flanks it: absolute standard identity is needed in order to express the modal thesis that there is inter-world identity, while relativized, contingent identity is needed in order to formulate CAI.

It is important to make clear from the beginning than we are not supporters of CCAI, or of any other version of CAI. We think and we have argued in other works ${ }^{1}$ that other, nonmodal problems affect CAI in its various forms and we do not think that CCAI alleviates these non-modal problems. Nonetheless, we think that CCAI contributes to show that modality is not the terrain on which CAl should be refuted. The extant literature already shows that CAI can be developed on the background of different views of modality, such as those endorsed by Bøhn, Borghini and Wallace. However, as far as we know, nobody tried to combine CAI with the Kripkean view of modality, a view which is implicitly assumed by several modal logicians and explicitly endorsed by some metaphysicians. Our purpose is to show that there is a credible version of CAI (CCAI) which is compatible with the Kripkean view of modality and that, as a consequence, this rather common view of modality is not incompatible with CAI.

We proceed as follows. In $\S 2$ we present the ways in which the relation between $\mathrm{NI}$ and CAI is analysed in the current literature. In $\S 3$ we present the broadly Kripkean view of modality on the background of which we shall develop CCAI. In $\S 4$, we show why, on the background of such a Kripkean view of modality, composition should be relativized to possible worlds, in order to avoid a conflict with the principle of the Indiscernibility of Identicals. In $\S 5$ we argue that the only way to make sense of CAI on the background of the broadly Kripkean view of modality we assume is to relativize also identity to possible worlds, and to formulate the resulting variety of CAI (CCAI) in terms of this relativized form of identity. In $\S 6$ we discuss the resulting distinction between the world-relative identity which

1 (Carrara \& Lando 2016, 2017), (Lando 2017, Appendix).. 
composition would be on the one hand, and standard identity on the other. In $\S 7$ we present an important objection to CCAl, according to which CCAI would be ad hoc. In $\S 8$, in order to deepen the analysis of this problem, we compare CCAI with other non-standard theories of identity, specifically Gibbard's theory of contingent identity (Gibbard 1975) and Gallois' theory of occasional identity (Gallois 1990, 1998). In $\S 9$ we compare CCAI with the extant varieties of CAI and we claim that CCAI should be classified as an interesting version of Weak CAl, according to which composition is not strictly speaking absolute identity, but is merely analogous to it. Finally, in $\S 10$ we draw some conclusions about how CCAl fares in comparison to other ways of addressing the modal problems of CAI.

\section{§ 2. Composition as Identity and the Necessity of Identity}

The interaction between $\mathrm{CAl}$ and $\mathrm{NI}$ can be presented in a seemingly simple way. By adopting even simpler formulations of CAI and $\mathrm{NI}$ than those adopted in $\S 1$, one could be tempted to argue as follows:

1) composition is identity (CAI);

2) identity is necessary ( $\mathrm{NI})$;

3) therefore, composition is necessary.

The Netherlands, Belgium and Luxembourg compose Benelux. Thus, according to $\mathrm{CAI}$, the Netherlands, Belgium and Luxembourg are identical to Benelux. According to $\mathrm{NI}$, any instance of identity holds necessarily. Thus, the relation that holds between Netherlands, Belgium and Luxembourg on the one hand and Benelux on the other (a relation that is an instance of identity, but also an instance of composition) holds necessarily. Thus, the Netherlands, Belgium and Luxembourg necessarily compose Benelux.

Consider another example: a chair $c$ is composed of four legs $\left(I_{1}, I_{2}, I_{3}, I_{4}\right)$, a seat $(s)$ and a back (b). Given CAI, $I_{1}, I_{2}, I_{3}, I_{4}, s$ and $b$ are identical to the chair $c$. According to $\mathrm{NI}$, every instance of identity holds necessarily. Thus, $I_{1}, I_{2}, I_{3}, I_{4}, s$ and $b$ are necessarily identical to $c$. The relation between $I_{1}, I_{2}, I_{3}, I_{4}, s$ and $b$ on the one hand and $c$ on the other holds necessarily. Thus, $I_{1}, I_{2}, I_{3}, l_{4}$, $s$ and $b$ necessarily compose $c$.

Observe that the necessity of composition also leads to the necessity of parthood, given some innocent mereological assumptions. Take the framework of classical extensional mereology (a framework which is widely assumed in the debate about CAI). Composition is simply the converse of mereological fusion: some entities $t$ compose an entity $u$ iff $u$ is the mereological fusion of $t$. Thus, it follows from the above reasoning, that, for any $x$ and $y y$, if $x$ is the fusion of $y y$, then $x$ is necessarily the fusion of $y y$ :

$$
\forall x \forall y y(x \Sigma y y \rightarrow \square x \Sigma y y) \quad \text { (Necessity of Fusion) }
$$

Given any plausible definition of mereological fusion, the fact that $x$ is the fusion of some $y y$ entails that any entity $z$ which is one of $y y$ is part of $x$. For example, the following, standard definition of fusion explicitly warrants this in the first conjunct of the definiens:

$$
x \Sigma y y \equiv_{\text {def }} \forall z(z \prec y y \rightarrow z P x) \wedge \forall z(z P x \rightarrow \exists w(w \circ z \wedge w \prec y y))
$$

(Fusion Definition)

As a result, whenever $x$ is the fusion of some $y y$ (that is, $x$ is composed of some $y y$ ), any $z$ which is one of $y y$ is necessarily part of $x$ (we will use ' $\prec$ ' for the plural logic relation is one of):

$$
\forall x(x \Sigma y y \rightarrow \forall z(z \prec y y \rightarrow \square z P x)) \quad \text { (Necessity of Being Part of a Fusion) }
$$




$$
\begin{aligned}
& \text { Now, consider (Plural Covering): }{ }^{2} \\
& \forall x \forall y(x P y \rightarrow \exists z z(x \prec z z \wedge y \Sigma z z)
\end{aligned}
$$

(Plural Covering)

(Plural covering) warrants that, in any case of parthood between a part $x$ and a whole $y$, there are some entities $z z$ such that the part $x$ is one of them and the whole $y$ is the fusion of them. (Necessity of Being Part of a Fusion) holds for any fusion and claims that every part of this fusion is necessarily part of it. Thus, given a standard mereological framework and the seemingly innocuous (Plural Covering), we obtain that any instance of parthood holds necessarily. This result is usually labelled as mereological essentialism. Here we will call it (Necessity of Parthood): ${ }^{3}$

$$
\forall x \forall y(x P y \rightarrow \square x P y)
$$

(Necessity of Parthood)

According to the foes of CAI (such as (Merricks 1999)), composition and parthood are plainly contingent. As a consequence, composition lacks a constitutive feature of identity, and the fact that composition lacks it would prove that composition is not identity, thereby refuting CAI. Thus (Merricks 1999) chooses the option a) among the options we listed in $\S 1$.

In the simple argument at the beginning of this section, 3) (the necessity of composition) is unacceptable, inasmuch as it contradicts $\mathrm{CC}$, a plain truth: every concrete entity could have different parts. Thus, one of the two premises of the simple argument should be rejected. However, 2) might seem unassailable, insofar as necessity is usually deemed a constitutive feature of identity. As (Barcan 1947) and (Kripke 1971) have shown, the necessity of identity follows from the Necessary Reflexivity of Identity and from the Indiscernibility of Identicals. In $\S 1$ we have presented an instance of this argument, concerning Donald Trump. Here is the argument in his general form:

$$
\begin{array}{lr}
\forall x \square x=x & \text { (Necessary Reflexivity of Identity) } \\
\forall x \forall y(x=y \rightarrow \forall P(P x \leftrightarrow P y)) & \text { (Indiscernibility of Identicals) }
\end{array}
$$

$$
\forall x \forall y(x=y \rightarrow \square x=y)
$$

(Necessity of Identity - NI)

If 2) ( $\mathrm{NI})$ is unassailable in the light of the Barcan-Kripke argument, then the falsity of 3 ) (the necessity of composition) forces us to reject 1 ): composition is not identity.

On the other side of the dialectics, the friends of CAI have reacted to Merricks' challenge in one of the following two ways. Some backers of CAI (Bøhn 2009; Borghini $2005)^{4}$ chooses the option b) in the list of $\S 1$. Namely, they endorse counterpart theory, in which identity is allowed to be as contingent as composition. Given counterpart theory, b)

2 See for example (Sider 2014, p. 212).

3 The label "mereological essentialism" comes from (Chisholm 1976). However, the essentiality at stake was meant simply as a necessity de re, without any commitment to the idea that parts are, as a consequence of any of the arguments we are discussing, essential in a stronger sense, namely in the sense of grounding what a whole is. This stronger understanding of essence-related terminology has become common (again) following (Fine 1994). For this reason, we will henceforth refrain from speaking of mereological essentialism.

4 In (Borghini 2005) there is not - strictly speaking - a defence of CAl, inasmuch as Borghini's focus is on counterpart theory. Nonetheless, as a matter of fact, Borghini shows that CAI can be made compatible with CC, provided that various versions of the theory of counterparts are adopted. It should be noted that (Merricks 1999) also admits that counterpart theory can make CAl compatible with the contingency of composition and parthood, but limits this admission to a specific version of counterpart theory. Nonetheless, Merricks is a foe of CAl, since he thinks that counterpart theory is a wrong analysis of modality (Merricks 2003). 
$(\mathrm{NI})$ is rejected. The fact that composition is contingent does not show that composition is not identity, insofar as identity is contingent as well.

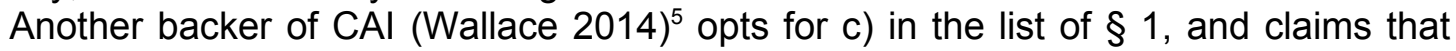
composition and parthood are indeed as necessary as identity, and that (Necessity of Composition) and (Necessity of Parthood) are independently plausible, when they are conjoined with a doctrine of modal parts. Entities would have necessarily all their parts, including their modal parts: Trump would necessarily have a modal part in another possible world, which has undergone an additional hair transplant. The motivations laying behind CC would be satisfied by these modal parts: composition and parthood are necessary, but some special parts (modal parts) make entities different in different possible worlds.

We are not going to raise any objection against b) and c). We only aim to explore in the next sections another variety of CAI, CCAI, which aims to be compatible both with $\mathrm{NI}$ and with CC.

CCAI distinguishes two varieties of identity. One of them is standard, absolute identity, which is as necessary as the Barcan-Kripke argument shows it to be. The other variety of identity countenanced by CCAI is contingent. Thus, the necessity of standard identity $(\mathrm{NI})$ is preserved. Any instance of composition is an instance of the second, contingent variety of identity, thereby preserving also CC. CAI, NI and CC turn out to be mutually consistent, in contrast with the assumption shared by a), b) and c) in the list of $\S 1$.

How to obtain this result? We shall argue that CCAI is the only way to make sense of CAI, once a Kripkean view of modality, and in particular the thesis that there is inter-world identity, is assumed. Let us begin by presenting the most salient traits of this view of modality.

\section{§ 3. Inter-World Identity, Indiscernibility and Relativization}

Prominent philosophers such as Kripke, Plantinga, Stalnaker and recently Williamson ${ }^{6}$ agree that there is identity across possible worlds (or inter-world identity). Individuals exist at possible worlds. While these authors disagree about the nature of possible worlds, they share the view that there is inter-world identity. There are many different possible worlds, corresponding to maximal and consistent ways in which things might be. Some of them are such that the same entity exists at all of them. Many maximal and consistent possible ways in which things might be are such that, for example, Donald Trump would exist if things were in that way: at some of these possible worlds Trump is married with Michelle LaVaughn Robinson, while at some others (including the actual world @) he is not married with Michelle. At some of them Trump is an ardent Democrat, while at some others (including @) he is not. At some of them Trump is 5 feet tall, while at some others (including @) he is not 5 feet tall (at @ he is 6 feet 2 inches). Trump exists at all these possible worlds.

Identity is standardly construed as that relation which every entity bears to itself and to nothing else. The claim that one and the same entity exists at various possible worlds immediately leads to the claim that there is identity across different possible worlds. Donald Trump - the same individual - exists at several different possible worlds. Also $c$, the chair in our example above, and all the other entities involved in our examples, exist at several possible worlds.

Is there a problem of inter-world identity? The standard, Kripkean answer to the question is 'no'. Those who argue for the problematicity of the notion of inter-world identity do so on the grounds that we cannot make judgments of identity across worlds unless we have a criterion for inter-world identity. So, if we judge that someone in an alternative world is identical to Trump (to the chair $c$ ) we would need a criterion of identity saying under what conditions a person $p$ (a chair $c$ ) in a world $w$ is identical to the person $p^{\prime}$ (chair $c^{\prime}$ ) in a world $W_{1}$. But at the end of the day it is rather difficult to find such a criterion. Kripke's reply is that,

5 On the same topic, see also (Wallace 2009, Chapter 4).

6 (Kripke 1980; Plantinga 1974; Stalnaker 2003). In (Williamson 2013), not only entities are allowed to exist at more than one possible world, but every entity exists at every possible world. 
even if it is very difficult to find a set of necessary and sufficient conditions for identity, it does not follow that the notion of inter-world identity is illegitimate. The qualms on inter-world identity are due to a certain picture on the nature of possible worlds understood as "a distant country" (1980, p. 44). Possible worlds are stipulated, not discovered. And it is perfectly right to stipulate them as including an actually existing particular, which - as a consequence exists at more than one possible world.

We are not assuming such a view as intrinsically correct. However, it is a rather common stance about modality: thus, it is interesting to know what follows from the Kripkean view about CAI and whether - in particular - an interesting form of CAI can result from adopting it.

Let us therefore focus on a feature of the Kripkean view which bears some significant consequences for mereological relations and for CAI: the need to relativize standard properties and relations to possible worlds. One inter-world identity is taken on board, a well known problem with the principle of the Indiscernibility of Identicals ensues. The principle of the Indiscernibility of Identicals says that identical things share all their properties:

$$
\forall x \forall y(x=y \rightarrow \forall P(P x \leftrightarrow P y))
$$

(Indiscernibility of Identicals)

The problem ensues if we consider - for example - @, the actual world, at which Trump is not an ardent Democrat; and $w_{1}$, at which Trump is an ardent Democrat instead. Trump is identical to himself. However, being an ardent Democrat might seem to be a property that Trump both instantiates and fails to instantiate, in contrast with (Indiscernibility of Identicals), and even with the Principle of Non-Contradiction.

Luckily, the Kripkean view does not violate a solid principle such as (Indiscernibility of Identicals) and leads to no contradiction. This is because in the application of the apparatus of possible worlds the violation of (Indiscernibility of Identicals) is avoided by a form of relativization to possible worlds. Trump is 6 feet 2 inches tall at @ and is not 5 feet tall at @, but is 5 feet tall at $w_{1}$ and is not 6 feet 2 inches tall at $w_{1}$. In this way Trump (one and the same individual) instantiates both the property of being 6 feet 2 inches tall at @, and the property of being 5 inches tall at $w_{1}$. Since a similar relativization is applied to every standard property, no counterexample to (Indiscernibility of Identicals) ensues.

In the possible world semantics for modal languages, this does not usually require applying an index to predicate letters. What happens is that, for each predicate letter, the interpretation function of a Kripkean model structure assigns to each possible world a subset of the domain of that world. This subset includes the entities that instantiate at that world the property expressed by that predicate letter.

Thus, world $w_{1}$ has a certain domain, the set of the entities existing at that world. A subset $\mathrm{F}$ of this domain includes as elements only the entities that are 5 feet tall at $w_{1}$ (and so includes Trump as a member, in our example). For the predicate that expresses in the formal language the property of being 5 feet tall, the interpretation function assigns $F$ to $w_{1}$. The semantic treatment of predicative letters is radically different from the treatment of individual constants: in the case of individual constants, the interpretation function assigns to each world the same member of the union of the domains of all the possible worlds. By contrast, the extension of a predicate letter is allowed to vary from world to world, and in each world cannot go beyond the limits of the domain of entities existing at that world.

The formula in the formal language that corresponds to the English sentence "Trump is 5 feet tall" will have, as a consequence, different truth values at different worlds: the formula is true at a world $w$ iff the element of the union of the domains which the interpretation function associates to the constant for Trump is a member of the set which the interpretation function associates to the predicate for being 5 feet tall for $w$. Thus, the extensions of the predicates and the truth values of the formulas including those predicates are indeed relativized to worlds.

In what follows, we will not formulate the relativization as possible world semantics usually does. By contrast, we will make the relativization to worlds explicit in the object language, by marking the relativization of properties to worlds with an index for predicate 
letters: thus, " $D_{@}$ " is a predicate expressing the property of being an ardent Democrat at the actual world, while " $D_{w 1}$ " is a predicate expressing the property of being an ardent Democrat at $w_{1}$. The resort to indexes, besides being simpler than the explicit resort to model structures, underlines that we are directly interested in the fact that, in the context of the Kripkean view, for every entity $t$ there is no single property $P$ such that $P t$ and $\neg P t$. Nonetheless, it is important to keep in mind that the indexes are not meant to suggest that there are intensionally different features that are ascribed by predicates that differ by their index. The indexes merely signal that the extension of a certain predicate is allowed to vary from world to world.

\section{§ 4. World-Relative Mereological Relations}

What is the impact on mereology of the Kripkean view of modality we characterized in $\S 3$ ? Mereological relations should be relativized to worlds in the sense we have explained. It has no bearing on the need for this relativization that the predicates in mereology (such as $P$ for parthood, o for overlap, $\Sigma$ for fusion) be relational, binary predicates, while the predicates for properties such as being 5 feet tall or being an ardent Democrat are monadic predicates. The need for a relativization arises for relations as much as for properties, quite independently of any mereological consideration. London is to the north of Paris, but might be to the south of Paris. This means that in the actual world London is to the north of Paris and is not to the south of Paris, while in some other worlds London is to the south of Paris and is not to the north of Paris. This does not elicit any counterexample to (Indiscernibility of Identicals) in the Kripkean view of modality, because the relations of being to the south of and of being to the north of are relativized to worlds: this means that, for each world, there is a different set of ordered pairs which is the extension of the corresponding predicate.

The same happens in the case of mereological relations. There is a notebook in front of me on my desktop. Let us call it $b$. Consider $s$, the third sheet of paper in $b$. $s$ has been assembled (with the help of some glue) with the other sheets in $b$ at the paper mill. However, it is a contingent matter of fact that $s$ has been assembled in that way, instead of becoming part of another notebook, or instead of remaining unused due to the sudden bankruptcy of the paper mill. At @ $s$ is part of $b$. By contrast, at other worlds (say at $w_{1}$ ), $s$ is not part of $b$ :

$$
\begin{aligned}
& s P_{@} b \\
& \neg s P_{w 1} b
\end{aligned}
$$

The same holds for the leg $I_{2}$ and the chair $c$ in our previous example. It is contingent that $I_{2}$ is part of $c$, thus:

$$
\begin{aligned}
& l_{2} P_{@} c \\
& \neg l_{2} P_{w 1} c
\end{aligned}
$$

Also $\circ$ will be relativized. There is a magazine rack $r$ at my place. Thus, $f$, the furniture of my place, overlaps with the sum $s$ of all the magazine racks in the world. But it is clearly a contingent fact that I bought a magazine rack some months ago, thereby making it part of the furniture at my place and determining an overlap between $f$ and $s$. This does not make the magazine rack $f$ both overlap and fail to overlap with $s: f$ is not discernible from itself. At $@ f$ and $s$ overlap, but at other worlds they do not overlap.

$$
\begin{aligned}
& f \circ @ s \\
& \neg f \circ_{w 1} s
\end{aligned}
$$

As we have already remarked in $\S 2$, it is equally contingent that $c$ (the chair) is composed of $I_{1}, I_{2}, I_{3}, I_{4}, s$ and $b$ (the legs, the seat and the back), or - equivalently - that the 
fusion of $I_{1}, I_{2}, I_{3}, I_{4}, s$ and $b$ is $c$. There are worlds in which $c$ is composed of different parts, and there are worlds in which $b$ (for example) contributes to composing other chairs or different pieces of furniture. This does not make $c$ (or $b$ ) discernible from itself, because also composition and fusion should be relativized to worlds, as any other standard property or relation. In @ the fusion of $I_{1}, I_{2}, I_{3}, I_{4}, s$ and $b$ is $c$. There are other worlds (say $w_{1}$ ) in which the fusion of $I_{1}, I_{2}, I_{3}, I_{4}, s$ and $b$ is not $c$.

$$
\begin{aligned}
& c \Sigma_{@} l_{1}, l_{2}, l_{3}, l_{4}, s, b \\
& \neg c \Sigma_{w 1} l_{1}, l_{2}, l_{3}, l_{4}, s, b
\end{aligned}
$$

In a nutshell, in the context of a Kripkean view of modality, every mereological relation - including composition - should be relativized to worlds.

\section{§ 5. From World-Relative Fusion to World-Relative Identity}

Let us introduce CAI. CAI is the contention that composition is an identity relation. Thus CAI is about a relation - composition - which, in the context of the Kripkean view of modality and in order to preserve (Indiscernibility of Identicals), has to be relativized to worlds. CAI claims about a world-relative relation that it is identity.

The only way to make sense of CAl in this context is to assume that composition is a world-relative kind of identity. Suppose that we instead say that composition is an identity relation of the usual, absolute sort, which is not relativized to worlds. We would simply lose track of the relativization of composition to worlds. The chair $c$ is at @ the fusion of $s, b, l_{1}, I_{2}$, $I_{3}$ and $I_{4}$. By contrast, $I_{3}$ is not part at $w_{1}$ of $c$ and is replaced in it by another leg, $I_{5}$.

$$
\begin{aligned}
& c \Sigma_{@} s, b, l_{1}, l_{2}, l_{3}, l_{4} \\
& c \Sigma_{w 1} s, b, l_{1}, l_{2}, l_{5}, l_{4}
\end{aligned}
$$

Now, suppose that both this instance of $\Sigma_{@}$ and this instance of $\Sigma_{w 1}$ are instances of the common breed of identity: absolute, non world-relative identity. We would obtain that $c$ is absolutely identical both to $s, b, I_{1}, I_{2}, I_{3}, I_{4}$ and to $s, b, I_{1}, I_{2}, I_{5}, I_{4}$ :

$$
\begin{aligned}
& c=s, b, l_{1}, l_{2}, l_{3}, l_{4} \\
& c=s, b, l_{1}, l_{2}, l_{5}, l_{4}
\end{aligned}
$$

Identity is obviously an Euclidean relation, so we might conclude that:

$$
s, b, l_{1}, l_{2}, l_{3}, l_{4}=s, b, l_{1}, l_{2}, l_{5}, l_{4}
$$

This conclusion is unacceptable, and incompatible with the definition of plural identity:

$$
x x=y y \equiv_{d e f} \forall z(z \prec x x \leftrightarrow z \prec y y) \quad \text { (Plural Identity Definition) }
$$

$I_{3}$ is one of $s, b, I_{1}, I_{2}, I_{3}, I_{4}$, but is not one of $s, b, I_{1}, I_{2}, I_{5}, I_{4} ; I_{5}$ is one of $s, b, I_{1}, I_{2}, I_{5}, I_{4}$, but is not one of $s, b, I_{1}, I_{2}, I_{3}, I_{4}$. Thus, given (Plural Identity Definition):

$$
s, b, l_{1}, l_{2}, l_{3}, l_{4} \neq s, b, l_{1}, l_{2}, l_{5}, l_{4}
$$

Once we have relativized composition to worlds, it is simply wrong to construe CAI as the claim that composition is standard, absolute, non world-relative identity. One might obviously insist that CAl is the thesis that every instance of composition is an instance of standard, absolute, non world-relative identity. However, there is something odd in insisting in this context that composition is absolute, non world-relative identity. Given a world-relative 
relation, already prima facie it seems rather unwise to defend the thesis that that relation is an absolute, non-world-relative relation (such as standard identity). This move is going to clash with the reasons why we had relativized composition to worlds in the first place, thereby leading to unacceptable consequences.

One might also, at this point, claim that these unacceptable consequences should ignite a reductio of one of our assumptions, and in particular either of CAI or of the Kripkean view of modality. For example, (Merricks 1999) would proceed from these unacceptable consequences to a reductio of CAl: given $\mathrm{NI}$, identity is not world-relative, and thus composition is not identity. By contrast, (Bøhn 2014), (Borghini 2005) and (Wallace 2014) would proceed from these unacceptable consequences to a reductio of the Kripkean view of modality, and in particular of the claim that there is inter-world identity. However, if you think that the motivations in favour of CAl are solid, then Merrick's approach will be hardly desirable for you, and you will long for an alternative. By contrast, the approaches of Bøhn, Borghini and Wallace are going to please whoever thinks that there are independent reasons to replace the Kripkean view with an alternative view of modality, such as counterpart theory or the theory of modal parts: for these philosophers, these unacceptable consequences are simply symptoms of a wrong overall approach to modality. However, if you do not think that there are these independent reasons to reject the Kripkean view, then the move of Bøhn, Borghini and Wallace will be methodologically dubious to you, and you will long for an alternative: CAI is a highly controversial thesis and it would be reckless to attribute to it alone the dialectical burden of grounding a reductio of the widely adopted (and comparatively less controversial) Kripkean view of modality.

In both cases, the alternative you are longing for is a variety of CAl which is compatible with the Kripkean view of modality. The alternative is to claim that world-relative composition is an unusual sort of identity, namely world-relative identity: the second variety of identity to which CCAI is committed. According to this alternative, all the instances of a world-relative relation (composition) are instances of another world-relative relation (worldrelative identity).

CCAI does not lead to the problem discussed above: $\Sigma_{@}$ and $\Sigma_{w 1}$ are not the same identity relation, but two different world-relative identity relations (that is, ones with a different extension), $=_{@}$ and $=_{w 1}$ :

$$
\begin{aligned}
& c={ }_{@} s, b, l_{1}, l_{2}, l_{3}, l_{4} \\
& c={ }_{w 1} \quad s, b, l_{1}, l_{2}, l_{5}, l_{4}
\end{aligned}
$$

The world-relative relations $=_{@}$ and $=_{w 1}$ will both be plausibly Euclidean, but inasmuch as they are relativized to different worlds - there is no reason to infer that $s, b, l_{1}$, $I_{2}, I_{3}, I_{4}$ are identical to $s, b, I_{1}, I_{2}, I_{5}, I_{4}$, in contrast with (Plural Identity Definition). The inference is blocked by the relativizations to worlds. What happens is that, in each world, entities are identical to their parts. They are allowed to have different parts, and as a result they are identical to different things in different worlds. This conclusion is now acceptable, and compatible with the definition of plural identity.

At this point we are ready to formulate CCAl:

$$
\forall w \forall x \forall y y\left(x \Sigma_{w} y y \rightarrow x={ }_{w} y y\right)
$$

\section{§ 6. World-Relative Identity versus Standard Identity}

CCAI distinguishes two varieties of identity: standard, absolute identity and world-relative identity. CCAl is formulated, as we have seen, in terms of the latter, but the former is required by the Kripkean view of modality on the background of which CCAl emerges as the only way to make sense of CAI. 
Let us explain why it is not possible to get rid altogether of absolute, standard identity and replace it with world-relative identity. Standard identity is used to express the pivotal claim of the Kripkean view according to which there is inter-world identity. Inter-world identity is nothing else than standard, absolute identity. It is expected to hold between any entity and itself irrespectively of the world we are considering. So, for example, Trump exists at many possible worlds and he is self-identical exactly in the same way at all these worlds. Not only that, but in the Kripkean possible world semantics Trump is self-identical also in the worlds at which he does not exist. This is because the identity predicate is special: its usual semantic interpretation reflects the fact that it has a notorious connection with logic.

In $\S 3$ we have seen that, in order to preserve (Indiscernibility of Identicals), every standard property and relation has to be relativized to worlds, where this relativization simply corresponds to the fact that the interpretation function in a model structure is such that the extension of the corresponding predicate is allowed to vary from world to world. But identity is different: the identity predicate is not treated on a par with other predicates in Kripkean possible world semantics. The identity predicate is treated in a special way not only in Kripkean possible world semantics, but already in the semantics for first-order logic with identity. The way in which identity statements are interpreted is not by resorting to an extension of the identity predicate. The identity predicate is, from a syntactical viewpoint, a binary relational predicate. However, the truth conditions of identity sentences do not have the usual set-theoretic structure of the truth conditions of sentences where other binary relational predicates occur. In other words, the identity sentences are not said to be true iff the ordered pair of the referents of the two terms surrounding the identity predicate is a member of the set of ordered pairs which is the extension of the predicate. By contrast, in the standard semantics of first-order logic with identity, an identity sentence is said to be true iff the referents of the two terms surrounding the identity predicates are coreferential. ${ }^{7}$

Thus, the truth value of an identity sentence - already in the first order logic with identity - depends only on the referents of the referential expressions in it, without any role for the extension of the identity predicate. The way in which the interpretation function works for referential expressions and, in particular, for individual constants is a characterizing feature of the Kripkean view of modality. As we have already seen in $\S 3$, the referent of an individual constant is not allowed to vary from world to world, in sharp contrast with the extension of a predicate. The interpretation function for individual constants assigns the same individual to each world. This individual has not to be chosen exclusively from the domain of that specific world. By contrast, it can be any member of the set-theoretical union of the domains of all the worlds in the model structure.

As a consequence, the truth value of an identity sentence does not vary from world to world. This is the way in which Kripkean possible world semantics encodes NI: given how identity sentences are interpreted, there is no way in which the truth value of an identity sentence could vary from world to world. Thus, if an identity statement is true at a possible world (e.g. at the actual world @), then it is true at every possible world. ${ }^{8}$

Inter-world identity is simply the relation between Trump and Trump himself, between Benelux and Benelux itself and between $I_{2}$ and $I_{2}$ itself. It is simply the relation which every entity bears to itself and to nothing else, or - equivalently - the smallest reflexive relation on the universal domain. It does not matter from the viewpoint of which world we look at it.

7 (Carrara and Lando 2016) discusses the impact of the special semantic treatment of the identity predicate on CAI.

8 When we pass from first-order logic to plural logic, there is another predicate which is arguably as special as the identity predicate, namely "is one of" $(<)$. The semantic treatment of $<$ might be similar to that of identity in not resorting to the extension of $<$ : a sentence such as " $t<u u$ " is true iff the referent of " $t$ " is one of the referents of " $u u$ ". This semantic treatment of $<$ mirrors the prevailing idea that $<$ is as necessary as identity ( $<$ is strictly connected to plural identity, see (Plural identity Definition) above). This idea is defended by (Williamson 2010) and (Linnebo 2016). See (Uzquiano 2011) for some objections. (Hewitt 2012) has explicitly defended the minority thesis that $<$ is contingent. 
Thus, we cannot get rid of standard identity, which obeys (Indiscernibility of Identicals). The relativizations of all the properties and relations (with the notable exclusion of standard identity itself, as we have seen above) is motivated precisely by the need to preserve (Identity of Indiscernibles) for standard identity. There is little doubt that standard identity respects (Indiscernibility of Identicals). Suppose that for an entity $t$ there is a property $P$ such that $t$ instantiates $P$ and fails to instantiate $P$ :

$$
\exists P(P t \wedge \neg P t)
$$

Suppose that this property is $S$. We would obtain a contradiction:

$$
S t \wedge \neg S t
$$

Thus, denying (Indiscernibility of Identicals) for standard identity directly leads to a contradiction. The relativization of properties and relations we have discussed in $\S 3$ is indeed motivated by the need to preserve this basic principle from modal counterexamples.

By contrast, consider the kind of world-relative identity postulated by CCAI. As we have seen above, it cannot replace standard identity, but can only flank it. How does worldrelative identity work? A trivial difference between standard identity and world-relative identity is that the former is not world-relative, while the latter is. But a deeper difference concerns indiscernibility. (Indiscernibility of Identicals) for world-relative identity could be formulated with a universal quantification over worlds, without any restriction on the properties at stake: ${ }^{9}$

$$
\forall w \forall x x \forall y y\left(x x={ }_{w} y y \rightarrow \forall P(P x x \leftrightarrow P y y)\right)
$$

(Absolute Indiscernibility of World-Relative Identicals)

However, (Absolute Indiscernibility of World-relative Identicals) is false, and its falsity marks the crucial difference between world-relative identity on the one hand and standard identity on the other. World-relative identicals are discernible, and in particular modally discernible. $c$ (our chair) is the fusion of $s, b, I_{1}, I_{2}, l_{3}, I_{4}$ at @. According CCAI $c$ is identical to $s, b, I_{1}, I_{2}, I_{3}, I_{4}$ at @.

$$
c=@ s, b, l_{1}, l_{2}, l_{3}, l_{4}
$$

However, it is possible, as we have seen above, that $l_{3}$ is no part of that particular chair, and that another leg, $I_{5}$, takes its place. Consider the world $w_{1}$ where this happens. $c$ obviously is the fusion of $c$ at $w_{1}$ (inasmuch as being the fusion of at $w_{1}$ is a reflexive relation). By contrast, $c$ is not the fusion of $s, b, I_{1}, I_{2}, I_{3}, I_{4}$ at $w_{1}$.

$$
\begin{aligned}
& c \Sigma_{w 1} c \\
& \neg c \Sigma_{w 1} s, b, l_{1}, l_{2}, l_{3}, l_{4}
\end{aligned}
$$

This makes $c$ on the one hand and $s, b, I_{1}, I_{2}, I_{3}, I_{4}$ on the other discernible. The property of being such that $c$ is their fusion at $w_{1}$ makes $c$ discernible from $s, b, I_{1}, I_{2}, I_{3}, I_{4}$ : the former instantiates it, the latter do not. We might express this differentiating property through a plural lambda-abstractor in the following way:

$$
\lambda x x\left(c \Sigma_{w 1} x x\right)
$$

9 Henceforth, we will formulate the indiscernibility principles for world-relative identity with plural quantifiers: as it is usual in plural logic (see for example (Oliver and Smiley 2013, pp. 106-108)), plural variables can take both singular and plural values. In the typical case involved in CCAI, one of the relata of world-relative identity would be a single whole, while the other relatum would be many parts. 
This property is one among many counterexamples to (Absolute Indiscernibility of World-Relative Identicals). Any case of contingent composition will elicit similar counterexamples: the whole will be discernible from its parts (while being world-relatively identical to them), insofar as the whole is the fusion of itself at every world at which it exists, while at some worlds the whole is not the fusion of those parts.

World-relative identity obeys a different indiscernibility principle. In particular, a backer of CCAI will want to claim that world-relative identity obeys a principle which - in contrast with (Absolute Indiscernibility of World-Relative Identicals) - does not state that world-relative identicals share all their properties (including those properties which are relative to other possible worlds), but only says that world-relative identicals share the properties relativized to that world. Such a restricted form of indiscernibility principle might be expressed as follows:

$$
\forall w \forall x x \forall y y\left(x x={ }_{w} y y \rightarrow \forall P\left(P_{w} x x \leftrightarrow P_{w} y y\right)\right)
$$

(World-Relative Indiscernibility of World-Relative Identicals)

Informally, for every world $w$, for every plurality $x x$ and for every plurality $y y$, if $x x$ are identical to $y y$ at $w$, then, for every property $P, x x$ instantiate $P$ at $w$ iff $y y$ instantiate $P$ at $w$. World-relative identity is such that relative identicals are allowed to instantiate and to fail to instantiate one and the same property: the properties at stake (the sources of the discernibility of world-relatively identicals) are not relativized to the world at which the entities are identical. Nonetheless, they are genuine sources of discernibility. The admission of these sources of discernibility is enough to make world-relative identity different from standard identity. For standard identity, it is utterly unacceptable that there is a property or relation whatsoever (no matter what the property is relative to) such that standardly identical entities instantiate and fail to instantiate it. By contrast, this routinely happens with world-relative identity, the kind of identity which - according to CCAI - composition is.

\section{§ 7. The Charge of Adhocness}

Let us take stock. Three main features differentiate standard identity from the kind of worldrelative identity which the backer of CAI is forced to introduce in the context of the Kripkean view of modality.

On the one hand, standard identity:

a1) is not relative to a possible world;

a2) holds necessarily (NI); Identicals).

a3) is governed by an unrestricted principle of indiscernibility (Indiscernibility of

On the other hand, the kind of world-relative identity which characterizes CCAI:

b1) is relative to a possible world;

b2) does not hold necessarily;

b3) is governed by a restricted principle of indiscernibility, i.e. (World-Relative Indiscernibility of World-Relative Identicals), and does not obey (Absolute Indiscernibility of World-Relative Identicals), i.e. the unrestricted principle for world-relative identity that corresponds to (Indiscernibility of Identicals).

These differences determine both the success of CCAI and a risk for it. The success consists in preserving the contingency of composition (CC) and of all the other mereological relations, as well as the necessity of standard identity $(\mathrm{NI})$. This result can be obtained only by distinguishing two varieties of identity, the one necessary, the other contingent. 
On the other hand, the risk consists in the fact that the way in which world-relative identity is introduced risks being ad hoc. On the basis of what we have said so far, indeed, there does not seem to be any reason to think that there is a world-relative kind of identity, besides the need to defend CAI. To say that the relation of composition between Benelux, the Netherlands, Belgium and Luxembourg is an instance of identity relativized to the actual world does not seem to be a way to assimilate composition to an already known and independently characterized relation. CCAI is not a way of saying that composition is something else, already known. A foe of CCAl might even insinuate that "world-relative identity" is simply a new name for world-relative composition.

Composition, like any other standard property or relation, is relativized to worlds in the context of the Kripkean view of modality; and then CCAI tells us that world-relative composition and other cognate cases are world-relative identities. What is the content - the foe of CCAI might ask - of this claim? Does it really say something interesting about worldrelative composition and cognate cases? In order to assess this charge of adhocness, let us have a look at other applications of world-relative identity.

\section{§ 8. Contingent Composition as Identity and Other Doctrines of Contingent ldentity}

Let us compare CCAI with other extant doctrines of non-standard identity, and in particular with doctrines of contingent identity. Perhaps, these doctrines can point to other applications of world-relative identity, and show that CCAl is not the unique, ad hoc motivation for introducing it. We will compare CCAI with Gibbard's contingent identity (Gibbard 1975) and with Gallois' occasional identity (Gallois 1990, 1998). It is important to make clear that we are not going to motivate or to endorse explicitly or implicitly these theories: we simply look for other potential domains of application for CCAl's world-relative identity, while keeping in mind that this can alleviate the charge of adhocness raised against CCAl only if either Gibbard's theory or Gallois' theory is accepted as true.

Let us begin from Gibbard's contingent identity proposal. In his most famous example, there is a statue, Goliath, and a piece of clay constituting the statue, Lumpl. As a matter of fact, Goliath and Lumpl persist during exactly the same period of time. They are identical, but only contingently so: Lumpl might have persisted for a longer time, if the piece of clay were squeezed into a ball before having the chance to dry. Thus, Goliath is identical to Lumpl at the actual world, but there are other worlds (such as $w_{1}$ ) at which Goliath is not identical to Lumpl. In our language: ${ }^{10}$

$$
\begin{aligned}
& \text { Goliath }=@ \text { Lumpl } \\
& \text { Goliath } \neq_{w 1} \text { Lumpl }
\end{aligned}
$$

Gibbard does not adopt the Kripkean view of modality, which we have assumed in developing CCAI, but the Carnapian doctrine of individual concepts. ${ }^{11}$ Thus, the modal background is utterly different from ours and no solace from the charge of adhocness against CCAl is going to come from this aspect.

Nonetheless, there is a significant link between Gibbard's motivations for claiming that Goliath and Lumpl are identical at the actual world and mereology. This link suggests that it might make sense to apply world-relative identity (introduced above for the purposes of CCAI) to Goliath, Lumpl and analogous cases. Let us see why.

Gibbard indeed claims that the main motivation for claiming that Goliath and Lumpl are identical is that they are made of the same fundamental physical entities. More exactly,

10 In (Gibbard 1975, p. 193) these claims are expressed by employing operators such as "in w" instead of our indexes.

11 In particular, Gibbard makes reference to the version of this doctrine set forth in (Bressan 1972). 
Gibbard claims that they are the same "set" of fundamental physical entities. In Gibbard's words:

Suppose, for example, we take point-instants to be our fundamental physical entities, and let a concrete thing be a set of point-instants. In that case, Goliath = Lumpl simply because they are the same set of point-instants. (Gibbard 1975, p. 192)

It is easy to translate Gibbard's set-theoretic talk in mereological terms. The idea becomes that Goliath and Lumpl would be the fusion of the same entities at the actual world:

$$
\exists x x\left(\text { Goliath } \Sigma_{@} x x \wedge \text { Lumpl } \Sigma_{@} x x\right)
$$

Gibbard does not say explicitly why this is a reason to think that Goliath and Lumpl are identical at the actual world, but this reason might be given by a principle in the vicinity of Uniqueness of Fusion, an axiom in many presentations of classical mereology:

$$
\forall x \forall y \exists z z\left(x \sum z z \wedge y \Sigma z z \rightarrow x=y\right)
$$

(Uniqueness of Fusion)

Let us adapt (Uniqueness of Fusion) to the relativization to worlds of mereological relations and introduce in it world-relative identity. We obtain the following principle:

$$
\forall w \forall x \forall y \exists z z\left(x \Sigma_{w} z z \wedge y \Sigma_{w} z z \rightarrow x={ }_{w} y\right)
$$

(Uniqueness of Fusion - Relativized)

Given (Uniqueness of Fusion - Relativized), the claim that Goliath and Lumpl are fusions of the same fundamental physical entities at the actual world implies that Goliath and Lumpl are identical at the actual world.

It seems that we can easily find a place for CCAI in this picture. No mention of any forerunner of CAI is to be found in (Gibbard 1975). However, there is an apparent intimacy between, on the one hand, the reasons why, according to Gibbard, Goliath and Lumpl are contingently identical; on the other hand, the reasons why, in CCAI, a contingent, worldrelative variety of identity is introduced in order to make CAI compatible with the contingency of composition (CC).

Goliath and Lumpl might be different and are, as a consequence, only contingently identical. This difference typically consists in the fact that Goliath and Lumpl might have different parts at some times. For example, we can suppose that at $w_{1}$ Goliath is formed by molding, at a certain point, Lumpl with a different piece of clay, Lumpm. The portion of clay which constitutes Goliath at $w_{1}$ is Lumpn, the fusion of Lumpl and Lumpm. From the viewpoint of CCAI, this means that Goliath is:

i) composed at $w_{1}$ by different fundamental physical entities from those which compose Goliath at @;

ii) is not identical at $w_{1}$ to Lumpl (but to Lumpn, the fusion of Lumpl and Lumpm).

i) and ii) are grounded in a single difference between @ and $w_{1}$ : the different formation of Goliath determines both a difference between @ and $w_{1}$ with respect to the instances of composition involving Goliath and a difference between @ and $w_{1}$ with respect to the instances of identity involving Goliath. In this case (and in every other case in which both mereology and constitution are involved) the source of the contingency of identity according to Gibbard is also the source of the contingency of composition (CC) according to CCAI. This gives at least a prima facie reason to think that CCAl and Gibbard's contingent identity might be consolidated in a single framework. The application of the world-relative variety of identity to the relation between physical objects and pieces of matter would make the application of world-relative identity to CCAI less specific and ad hoc.

In spite of this affinity of motivations, it should be admitted that the perspective of integrating CCAI and Gibbard's contingent identity in a cohesive framework is clouded by a 
couple of important differences. First - as remarked above - Gibbard adopts a non-Kripkean view of modality, and he is, for example, simply silent on his view of possible worlds and inter-world identity. Second, Gibbard's contingent identity is said to obey Leibniz's Law in its absolute form, since - according to Gibbard, who makes reference to (Quine 1954) on this "expressions constructed with modal operators [...] simply do not give properties of concrete things, such as statues of pieces of clay" (Gibbard 1975, p. 201). Thus, modal formulas do not attribute properties, and there are - as a consequence - no modal properties in the domain of properties upon which the indiscernibility principle quantifies. Inasmuch as there are no modal properties, modal properties are no counterexample to (Indiscernibility of Identicals). Gibbard's contingent identity is therefore said to obey (Indiscernibility of Identicals). ${ }^{12}$

Gallois' doctrine of occasional identity is another promising term of comparison for CCAI and in particular a promising field of application for world-relative identity. This might not be immediately evident, insofar as Gallois' primary (but not exclusive, as we are going to see) concerns are time and change, topics which might be relevant for CAl, but are not the subject matter of CCAI and of this paper in general. A famous example of occasional identity in (Gallois 1990) is the following: at time t', John has its entire brain; later, the left half of John's brain is transplanted in John-Left, while the right half of John's brain is transplanted in John-Right. According to Gallois, at $t$ ' John-Left is identical to John-Right; however, at $t$ "' (a time after the transplantation) John-Left is not identical to John-Right. The relation in which John-Right and John-Left are at $t^{\prime}$ and are not at $t^{\prime \prime}$ is occasional identity.

Occasional identity does not respect an unrestricted principle of indiscernibility, but a restricted principle which is structurally similar to our (World-Relative Indiscernibility of World-Relative Identicals) above. We proceed as follows. First, let us see why occasional identity does not respect an unrestricted principle of indiscernibility, then we discuss the similarity between Gallois' restricted principle of indiscernibility and our (World-Relative Indiscernibility of World-Relative Identicals).

John-Right and John-Left are identical at $t^{\prime}$, in spite of having different properties at $t$ ": suppose for example that only John-Right is bald at $t$ ", while John-Left is not bald at $t$ ". This means that only John-Right - and not John-Left - is bald at $t$ ": thus, John-Right and John-Left are discernible, but their discernibility at $t$ " does not make them different neither at $t^{\prime}$ nor at any other time different from $t$ ".

The discernibility principle for occasional identity at a certain time $t$ is restricted to the properties instantiated at that time $t$. We could formulate the indiscernibility principle for occasional identity as follows: ${ }^{13}$

$$
\forall t \forall x \forall y\left(x={ }_{t} y \rightarrow \forall P\left(P_{t} x \leftrightarrow P_{t} y\right)\right)
$$

(Time-Relative Indiscernibility of Occasional Identicals)

This principle can be obtained from Gallois' correspondent of our (World-Relative Indiscernibility of World-Relative Identicals) by replacing the variable $w$ for worlds with the variable $t$ for times.

Moreover, (Gallois 1998, Chapter 6) develops a theory of contingent identity which strictly parallels his theory of occasional identity and proposes at a point (page 145) that contingent identity should follow a modal equivalent of (Time-Relative Indiscernibility of Occasional Identicals), which corresponds to our (World-Relative Indiscernibility of WorldRelative Identicals): ${ }^{14}$

12 These differences might perhaps be disposed of by modifying either CCAI or Gibbard's theory, but we are not going to pursue this perspective.

13 See for example (Gallois 1998, p. 81). Gallois opts for indicating times with an "at $t$ ". In our version of the principle we replace the operator with a time index (and a world index below), for the sake of consistency with our presentation of CCAI. Moreover, Gallois opts in his works for a schematic formulation of indiscernibility principles, while in this paper we always formulate indiscernibility principles with a second-order quantification over properties. 


$$
\forall w \forall x \forall y\left(x={ }_{w} y \rightarrow \forall P\left(P_{w} x \leftrightarrow P_{w} y\right)\right)
$$

Gallois also explains that such a principle cannot replace absolute (Indiscernibility of Identicals) in the Barcan-Kripke argument for $\mathrm{NI}:{ }^{15}$ the correspondent of our (World-Relative Indiscernibility of World-Relative Identicals) in Gallois' doctrine is a principle suitable for a contingent variety of identity.

Gallois, not differently from Gibbard in this respect, does not discuss any thesis akin to CAI. Nonetheless, Gallois makes some references to mereology, which are useful to assess the extent at which CCAI and Gallois' contingent identity might be integrated, thereby addressing the charge of adhocness raised against CCAI.

One of Gallois' examples of contingent identity ${ }^{16}$ concerns a car, whose name is "Car", and the fusion of its parts, whose name is "Car". As a matter of fact, Car and Car" are identical at every time, because they begin to exist and cease to exist at the same times and no part of Car is ever replaced. Nonetheless - Gallois argues - Car and Car apparently differ in their modal properties: only Car - and not Car* - might have lacked a right front wheel. The idea is that Car has a sort of mereological flexibility, which Car* lacks. ${ }^{17}$

Let us see if and how it is possible to insert CCAI in this picture. At the actual world, Car and Car* have the same parts (at every time, but we omit in what follows any mention of times, since they do not play any role in this example). " $c c$ " is a plural constant for these parts. At the actual world, according to CCAI, both Car and Car* are identical to these parts:

$$
\begin{aligned}
& \mathrm{Car}=@ c c \\
& \mathrm{Car}^{*}=@ c c
\end{aligned}
$$

It follows, from these instances of CCAI and from the fact that $=_{@}$ is left Euclidean, that the same relation expressed by " $=@$ " also connects Car and Car*:

$$
\text { Car }=@ \mathrm{Car}^{*}
$$

There is no reason to doubt that this relation is the same relation of contingent identity which - quite independently of CCAI - Gallois' doctrine countenances between Car and Car*. ${ }^{18}$

Let us deepen a bit this integration. Car - in contrast with Car* - might lack a right front wheel. $w_{1}$ is a possible world at which Car is different from Car*, according to Gallois. At $w_{1}$ Car is the fusion of $c c$, while Car* is not the fusion of $c c$ at $w_{1}$. Once we add CCAl to the picture, what happens is that Car is not identical to $c c$ at $w_{1}$, while Car* is identical to $c c$ at $w_{1}$.

14 A difference between this principle and our (World-Relative Indiscernibility of World-Relative Identicals) is that Gallois does not consider many-one or many-many cases of identity (and in general does not employ plural tools). As a consequence, in presenting his doctrines, we restrain from using plural variables, which are by contrast needed in (World-Relative Indiscernibility of World-Relative Identicals).

15 See in particular (Gallois 1998, pp. 160-165).

16 (Gallois 1998, p. 141).

17 See (Gallois 1998, sec. 5.IX) about the role of mereological (in)flexibility in Gallois' doctrine.

18 Please keep in mind that we are simply developing some consequences of the interaction of CCAI and Gallois' doctrine and claims. Gallois' claim that Car and Car* are the fusion of the same parts at @ may be questioned for a variety of reasons. For example, one might suggest that the reasons to distinguish Car and Car* at a certain world also apply to their parts; in general the reasons to distinguish an artifact from the piece of matter constituting it seem to concern at the same degree both the entire artifact (e.g. the car) and its parts, in particular its functional parts (such as the steering). This suggestion is developed in (Varzi 2008).

If you disagree with Gallois that Car and Car* are identical to the same fusion of parts, then this specific example will seem wrong to you, but this has no direct consequence on the perspective of integrating CCAI and Gallois' doctrine of contingent identity. 


$$
\begin{aligned}
& \text { Car } \neq_{w 1} c c \\
& \text { Car* }^{*}{ }_{w 1} c c \\
& \text { Car } \neq{ }_{w 1} \text { Car* }^{*}
\end{aligned}
$$

Thus, Gallois' doctrine of contingent identity seems to be expresible through that same world-relativized identity which CCAl needs. One might wonder whether also his doctrine of occasional identity might be analogously integrated with a temporal equivalent of CCAl: a form of Occasional Composition as Identity (OCAI). What might happen in the integration of Gallois' occasional identity and OCAI, in the example of John-Left and JohnRight above, would be that at $t$ ' (the time before the brain transplantation) John-Left and John-Right would be temporally/occasionally identical not only one to another, but also to their parts $j j$.

$$
\begin{aligned}
& \text { John-Left }={ }_{t^{\prime}} \text { John-Right } \\
& \text { John-Left }={ }_{t^{\prime}} . j \\
& \text { John-Right }=t_{t^{\prime}} . j
\end{aligned}
$$

By contrast, at $t$ " (the time after the split brain transplantation) John-Left is not identical to John-Right, and neither of them is the fusion of $j j$ : they have different parts (II are the parts of John-Left at $t^{\prime \prime}, r r$ are the parts of John-Right at $\left.t^{\prime \prime}\right)$ and are, according to OCAI, identical to these different parts at $t$ ".

$$
\begin{aligned}
& \text { John-Left } \neq_{t^{\prime \prime}} \text { John-Right } \\
& \text { John-Left } \neq_{t^{\prime \prime}} j j \\
& \text { John-Right } \neq_{t^{\prime \prime}} j j \\
& \text { John-Left }=t_{t^{\prime \prime}} l l \\
& \text { Johh-Right }=_{t^{\prime \prime}} \mathrm{rr}
\end{aligned}
$$

It is not possible to motivate and adequately develop OCAI in this paper (CCAI is complex enough for a single paper!). ${ }^{19}$ However, it should be noted that the comparison with Gallois' doctrine (in which occasional identity and contingent identity are analogously motivated and structurally similar) suggests that, in general, the claim that composition is a kind of non-standard, non-absolute, relativized identity might be harmoniously integrated with other, non directly mereological doctrines of non-standard, non-absolute, relativized identity.

While in the case of Gibbard the harmonious integration concerns at most the motivations (and not the way in which contingent identity is characterized), in the case of Gallois the harmonious integration concerns also the way in which relativized identity would work, in particular for what concerns the indiscernibility principle for relativized identity. All things considered, we can say that, at least for those who think that there is some merit to these theories of contingent identity, the charge of adhocness against CCAI is unjustified: world-relative identity has other fields of application.

\section{§ 9. Contingent Composition as Identity and Weak Composition as Identity}

CCAI, besides being charged of being ad hoc (the charge we have discussed in $\S 8$ ), might also be suspected of not being a genuine form of CAl. This suspicion might be presented as follows: CAl is the thesis that every instance of composition is an instance of identity, and

19 We plan to discuss OCAI in another work. The more direct competitors of CCAI are the combinations of CAI with counterpart theory and with the theory of modal parts (see $\S 2$ and $\S 10$ ). Analogously, the more direct competitors of OCAI are the combinations of CAI with exdurantism and perdurantism. As far as we know, neither of these combinations has been discussed in the literature about CAI. 
there is prima facie no evidence that the identity at stake in CAI is anything else than standard identity, which obeys an unrestricted principle of indiscernibility. Well, CCAI does not tell us that composition is this relation. Quite the opposite: CCAI clearly says that composition is another relation, which is governed by different principles. CCAI, from this viewpoint, risks being a denial of CAI, more than a version of it.

If, by stipulation, we establish that CAI is the claim that any instance of composition is an instance of standard identity, then indeed CCAI is not a variety of this claim, but its denial. Indeed, some backers of CAl construe it precisely in this way. For example, (Bøhn 2014) claims, in characterizing CAI, that "identity is the primitive relation everything bears to itself and to nothing else, uniquely characterized" by reflexivity and Leibniz's Law (p. 143). Indeed, CCAl is incompatible with Bøhn's thesis.

However, there are other versions of CAI and it is sensible to consider CAI as a family of theses, unified at most by the basic, generic contention that there is a significant affinity between composition and identity. After all, neither of the original supporters of the thesis in its contemporary form (Donald Baxter and David Lewis) would agree with Bøhn that composition is standard identity. Baxter ${ }^{20}$ does not adopt standard, Quinean identity at all: according to him, identity does not, in general, obey Leibniz's Law: identical entities are allowed to differ in properties and relations which are relative to so-called aspects. According to Lewis, ${ }^{21}$ identity is indeed the standard relation, uniquely characterized by reflexivity and by Leibniz's Law; but composition, while being analogous to this relation under several important respects, falls short of being it and does not obey an unrestricted principle of indiscernibility. In particular, a whole and its parts are discernible inasmuch as - for example - the whole is one and not many, while the parts are - typically - many and not one. Thus, there have been at least two prominent versions of CAl according to which composition is not standard identity, and the fact that CCAI does not claim that composition is standard identity does not disqualify CCAI from being regarded as a variety of CAI.

In order to explain positively why CCAI is a version of CAI and situate CCAI in the debate about CAI, it is useful to resort to a taxonomy of the various versions of CAI and to ask to which version of CAI CCAI is similar. There are several taxonomies in the literature. For the sake of simplicity, let us select one of them, namely the taxonomy proposed by (Wallace 2011a, sec. 2). ${ }^{22}$ Wallace distinguishes Weak CAI, Strong CAI and Stronger CAI.

1) According to Weak CAI, composition is not strictly speaking absolute, standard, Quinean identity, but is analogous to it. It is identity in a broadened sense. It does not obey some of the principles governing standard identity, and in particular the principle of the Indiscernibility of Identicals, but it is still similar to standard identity under highly significant respects. It is the variety of CAl set forth by one of the founders of CAI mentioned above - Lewis - and later by (Sider 2007).

2) According to Strong CAI, any whole is strictly identical to all its parts taken together and the identity at stake is standard identity, obeying an unrestricted principle of indiscernibility; it is the variety of CAI defended by (Bøhn 2014) and (Wallace 2011b).

3) According to Stronger CAI, composition is strictly speaking identity (in contrast with Weak CAI), but identity is not classically defined (in contrast with Strong $\mathrm{CAl}$ ), and in particular does not obey an unrestricted principle of indiscernibility. It is the variety of CAI set forth by the other founder of CAI mentioned above, Baxter.

20 (Baxter 1988a, 1988b, 2014).

21 (Lewis 1991, pp. 81-87).

22 Other taxonomies of the varieties of CAI are in (Cotnoir 2014; Sider 2007; Yi 1999). 
We are going to argue that CCAI is indeed better construed as a version of Weak $\mathrm{CAl}$, but let us begin by explaining why CCAI definitely cannot be a version of Strong CAI and bears only some similarities (as well as some deep differences) to Stronger CAI.

CCAI is plainly incompatible with Strong CAI, since - as we have seen - CCAl does not assimilate composition to standard identity and, in particular, claims that composition does not obey an unrestricted principle of indiscernibility, while Strong CAI precisely claims that composition is governed by an unrestricted principle of indiscernibility. By contrast, Weak CAI and Stronger CAI agree with CCAI (and disagree with Strong CAI) that composition does not obey the standard principle of the Indiscernibility of Identicals; thus CCAl is prima facie more compatible with them than with Strong CAI.

In discussing Stronger CAI we will follow the useful systematization of Baxter's theory in (Turner 2014). Stronger CAI is a piece of Baxter's wider revisionary theory of identity. Baxter is committed to two notions of identity: intra-count identity and cross-count identity. This kind of dualism about identity makes Stronger CAI prima facie similar to CCAI, which distinguishes two varieties of identity as well: standard identity and world-relative identity. However, a closer look to the two varieties of identity in Baxter's doctrine shows that the similarity with CCAI does not run deep. Let us first consider intra-count identity and then cross-count identity.

Intra-count Identity and CCAI. Baxter's intra-count identity concerns entities which belong to the same count, where a count is - roughly - a way to divide into distinct, countable entities the stuff reality consists in (Turner 2014, p. 230). Intra-count identity does not respect an unrestricted principle of indiscernibility, inasmuch as one and the same entity is allowed to be different from itself, due to the fact that the aspects of this entity determine this difference. In Turner's example, Jason is both a philosopher and a father. Jason spends a lot of time doing philosophy. Jason as a father (qua father) does too much philosophy, but it is not the case that Jason qua philosopher does too much philosophy. When aspects are involved (as in Jason's case), identicals are sometimes discernible. This means that intracount identity respects only a restricted form of indiscernibility principle, which roughly excludes properties depending on aspects. ${ }^{23}$

Intra-count identity does not really correspond to either of the two varieties of identity countenanced by CCAI. First, intra-count identity does not correspond to world-relative identity. Intra-count identity is, admittedly, superficially similar to the world-relative kind of identity which CCAI countenances, inasmuch as intra-count identity is, in a certain sense, relative to a count, as CCAl's world-relative identity is relative to worlds. However, in spite of this superficial similarity, there are two significant differences between CCAl's world-relative identity and Baxter's intra-count identity.

The first difference is that Baxter's intra-count identity is not the relation in terms of which Baxter's version of CAl is formulated. A whole and its parts typically belong to different counts: Benelux on the one hand and the Netherlands, Belgium and Luxembourg on the other are the results of different ways of dividing the same stuff, and thus belong to different counts. But intra-count identity is internal to a count, thus it cannot connect a whole and its parts: it does not connect Benelux with the Netherlands, Belgium and Luxembourg. By contrast, CCAl's world-relative identity is precisely expected to connect a whole and its parts: the identity claim about a whole and its parts is formulated by CCAI in terms of worldrelative identity.

The second difference is that the restriction on the indiscernibility principle for intracount identity does not consist in excluding from the domain of the second-order quantifier the properties relativized to a count, while in the case of CCAI the restriction in (WorldRelative Indiscernibility of World-Relative Identicals) consists in excluding from the domain of the second-order quantifier the properties relativized to a world. By contrast, the restriction for Baxter's intra-count identity excludes properties depending on aspects, and is in general

23 See (Turner 2014, p. 230) for a discussion of various ways of making precise this restriction of the indiscernibility principle for intra-count identity. 
required by Baxter's theory of aspects, a piece of his doctrine which - quite independently of its intrinsic merits - has no direct relation with CCAI. Thus, the restriction of the indiscernibility principle for intra-count identity is not similar to (World-Relative Indiscernibility of World-Relative Identicals), the principle of indiscernibility for CCAl's world-relative identity.

Second, intra-count identity does not correspond to CCAl's standard identity either, because intra-count identity does not obey an unrestricted principle of indiscernibility, while CCAl's absolute identity obeys an unrestricted principle of indiscernibility. Thus, intra-count identity does not correspond to either of the varieties of identity countenanced by CCAI.

Cross-count Identity and CCAI. The second variety of identity in Baxter's doctrine is cross-count identity. Cross-count identity typically connects entities which belong to different counts. It is the variety of identity in terms of which Baxter's variety of CAI is formulated. Baxter thinks indeed that the Netherlands, Belgium and Luxembourg are distributively cross-count identical to Benelux, and that - as a consequence - each of the three countries is individually cross-count identical to Benelux. This is analysed in terms of occupation of space regions: an entity $x$ is cross-count identical to an entity $y$ iff there is a space region $R$ such that $x$ exactly occupies $R$ and $y$ pervades $R$. That $x$ exactly occupies $R$ roughly means that $x$ is entirely at $R$ (there is no other region at which $x$ is). That $y$ pervades $R$ means that $y$ fills $R$ and perhaps more besides. ${ }^{24}$

There is also a many-one variety of cross-count identity. Some entities $x x$ are crosscount identical to $y$ iff each entity which is one of the $x x$ is individually cross-count identical to $y$. Thus, the Netherlands, Belgium and Luxembourg, which compose Benelux, are many-one cross-count identical to Benelux, inasmuch as each of the three countries is individually cross-identical to Benelux. In general, in any case of composition the composing entities are cross-count identical to the composed entity. This is the point at which Baxter's complex doctrine finally qualifies as a variety of CAI, since it claims that any instance of composition is an instance of (cross-count) identity.

Baxter's cross-count identity obeys a restricted indiscernibility principle. The details of this restriction are rather complex (see (Baxter 1988a, p. 208; Turner 2014, p. 236) for some discussion) and do not matter for the comparison with CCAI. As a very rough approximation, an individual part and a whole are expected to share any property that does not depend on counts (that is on the ways in which a portion of reality is divided in entities) or aspects.

Since cross-count identity (and not intra-count identity) is used in Baxter's formulation of CAI, one might hope to find a connection between it and CCAl's world-relative identity, in terms of which CCAI formulates its own claim that any instance of composition is an instance of identity. However, this hope would be ungrounded, for at least the following three reasons. First, counts and worlds have no interesting mutual connections: different counts are different ways of dividing the same stuff or the same portions of reality in entities; they are not alternative ways in which reality might be (as possible worlds are), but different ways in which the same actually existing stuff is divided. Second, CCAl's world-relative identity is with respect to worlds exactly the opposite of what Baxter's cross-count identity is with respect to counts: world-relative identity is confined to a single world, while cross-count identity connects entities in different counts. Third, CCAl's world-relative identity is not expected to hold between each individual part of a whole and the whole (as Baxter's crosscount identity does), but only collectively between the parts and the whole.

Finally, Baxter's cross-count identity does not correspond to CCAl's absolute, standard identity, inasmuch as the former does not respect an unrestricted principle of indiscernibility, while the latter does.

Thus, the affinity between CCAI and Stronger CAI does not go beyond the fact that both doctrines are committed to a form of dualism about identity. At the end of the day, the distinctions between two varieties of identity respectively drawn by CCAI and stronger CAI

24 (Turner 2014, p. 235) borrows this terminology from (Parsons 2007). 
are motivated by different needs, and the resulting varieties of identity are governed by different principles.

The last (and most promising) version of CAI at which to look for similarities, which might make CCAl a legitimate form of CAI, is Weak CAl: the variety according to which composition is not strictly speaking standard identity, but is significantly analogous to it, and is therefore identity in a broadened sense. The general ratio of a comparison between CCAI and Weak CAI is that CCAl claims that composition is world-relative identity, and worldrelative identity is not standard identity, but is significantly analogous to it. Thus, through the mediation of world-relative identity, CCAl ends up being the claim that composition is not standard identity, but is analogous to standard identity: it ends up being a form of Weak CAI.

What significant analogy is there, according to CCAI, between composition and standard identity, through the mediation of world-relative identity ${ }^{25}$ The analogy concerns the indiscernibility principles they obey. CCAI claims that world-relative identity obeys (World-Relative Indiscernibility of World-Relative Identicals). (World-Relative Indiscernibility of World-Relative Identicals) is not (Indiscernibility of Identicals), the unrestricted principle of indiscernibility which governs standard identity. Yet, it is not much different: it excludes only the properties relativized to worlds different from the world at which composition and worldrelative identity hold.

Consider the instance of contingent identity which holds at @ between the chair and its parts:

$$
c=@ l_{1}, l_{2}, l_{3}, l_{4}, s, b
$$

The chair and its parts at @ are only required to instantiate the same properties at @. It does not matter (it is not in contrast with (World-Relative Indiscernibility of World-Relative Identicals)) that - say $-I_{1}, I_{2}, I_{3}, I_{4}, s$ and $b$ do not compose the chair $c$ at another world $w_{1}$ (an instance of the contingency of composition) or the fact that - say - the chair $c$ is black at $w_{1}$, while $I_{1}, I_{2}, I_{3}$ and $I_{4}$ are blue at $w_{1}$ and $s$ and $b$ are white at $w_{1}$ (an instance of the contingency of non-mereological properties, which should be relativized to worlds as well in the Kripkean view of modality assumed in CCAI).

The affinity between (World-Relative Indiscernibility of World-Relative Identicals) and (Indiscernibility of Identicals) constitutes a significant analogy between world-relative identity and standard identity, thereby bringing forward the cause of Weak CAl. Although not unrestricted, (World-Relative Indiscernibility of World-Relative Identicals) is indeed an inclusive and ambitious principle. Thanks to it, composition and cognate relations are indeed analogous (as weak CAI contends) to standard identity, which obeys an even stronger indiscernibility principle, namely unrestricted (Indiscernibility of Identicals). This is the reason why CCAI can be sensibly seen as a form of Weak CAI, a version of CAI endorsed by one of the original backers of CAI, Lewis. Thus, CCAI deserves to be considered a variety of CAI.

\section{§ 10. Conclusions}

It is now time to conclude by assessing some virtues and some limits of CCAI. CCAI is a variety of CAI which - by adopting a broadly Kripkean view of modality - succeeds in preserving the contingency of composition (CC) and the necessity of standard identity (NI). This does not mean that CCAI is true.

25 The analogy we are going to discuss is independent from the analogies discussed by Lewis in his original presentation of Weak CAI (Lewis 1991, pp. 85-86) and our claim is not that Lewis would accept the analogy we discuss.

Lewis discusses five analogies between composition and identity. These analogies concern respectively - ontological innocence, unrestricted composition, uniqueness of composition, the ease of describing fusions if you have already described the fused entities, and the necessary colocation of a whole and its parts. Many of these analogies are strictly dependent on Lewis's doctrines about mereology, and each of them is highly controversial. See (Carrara and Martino 2011) for various objections to Lewis's claims. 
First, CCAI is simply limited to the modal aspects of composition and identity and does not address in any way the non-modal objections which have been raised against CAI and might be rehearsed against CCAI. To exemplify: CCAl adopts the ambitious (WorldRelative Indiscernibility of World-Relative Identicals), and this principle - as we have seen in $\S 9$ - is pivotal in justifying the ambition of CCAI to be a variety of CAl. However, (WorldRelative Indiscernibility of World-Relative Identicals) might be false for reasons which have nothing to do with modality: for example because cardinality ascriptions or other kinds of predications falsify it. Lewis himself (the main backer of Weak CAI) would object to (WorldRelative Indiscernibility of World-Relative Identicals) that the chair $c$ is one and not six at @, while $I_{1}, I_{2}, I_{3}, I_{4}, s$ and $b$ are six and not one at @. In order to answer this objection, the defender of CCAI would need to borrow from other varieties of CAI the strategies for relativizing cardinality ascriptions to concepts or ways of counting, and it is controversial that these strategies work. ${ }^{26}$

Second, even if we lay the non-modal problems of CAI aside, the problem remains whether CCAl is any better of its competitors on the modal terrain. CCAl should be compared to other ways of avoiding (Merricks 1999)'s attempt to refute CAI on the basis of $\mathrm{NI}$, such as the adoption of counterpart theory in order to reject $\mathrm{NI}$ in (Bøhn 2009) and (Borghini 2005) and the adoption of the theory of modal parts in order to make the rejection of CC acceptable in (Wallace 2014).

We have seen in $\S 8$ that the charge of adhocness against CCAI can be answered by showing that other doctrines of contingent identity point to other applications of world-relative identity, the non-standard variety of identity countenanced by CCAI. However, this puts CCAl at most on a par, from this viewpoint, with its competitors on the modal terrain, but not above them. Indeed, several doctrines apply counterpart theory to various philosophical problems (quite independently of $\mathrm{CAl}$ ), so that the adoption of counterpart theory may not be sensibly charged of being ad hoc. Also the theory of modal parts, while being less common, has been sometimes adopted for reasons independent of the need to defend CAl. ${ }^{27}$

A comparative difference among the three alternatives approaches to the modal aspects of CAl might consist in the ability of only one or two of them to solve other, nonmodal problems of CAI. However, the only extension to other problems of CAI which comes to our mind concerns time. We have indeed suggested that CCAI might be extended to a theory of occasional composition as identity, OCAl. But also the theory of counterparts and the theory of modal parts have some obvious extensions in the temporal domain, so that also in this case no alternative seems to gain an edge over the others.

It seems to us that, at the end of the day, the choice among CCAI and its two competitors mainly depends on the view of modality adopted. The most virtuous order in which one should proceed is: first, choose the best view of modality; second, if you are convinced by CAl's general motivations, develop CAI in a way compatible with the best view of modality. Inasmuch as CAI is a controversial thesis, which can be developed in a variety of ways, it would be methodologically unwise to proceed in the reverse order, i.e. to choose the best version of $\mathrm{CAl}$ and adopt a certain view of modality in order to accommodate it.

It is not the ambition of this paper to decide which is the best view of modality. The Kripkean view on the basis of which we have presented CCAl enjoys a primacy in modal logic. In particular, the thesis that there is inter-world identity is embodied in the fact that model structures (the most common semantical tool for modal logic) do not exclude that the domains of different worlds overlap. However, we have no inclination to think that philosophers should look to what is more common in logic in order to choose the best view of modality. Our ambition is more modest: we hope to have shown that CAI is not only compatible with counterpart theory and the theory of modal parts, as the existent literature had already shown, but also with the view that there is inter-world identity. CAl is not refuted by modal considerations and does not force you to adopt a different view of modality. By

26 See (Bøhn 2014; Cotnoir 2013; Hovda 2005; Wallace 2011b) for these strategies and (Carrara and Lando 2017) for a recent critical analysis.

27 (Graham 2015; Schlesinger 1985; Torrengo 2011; Varzi 2001). 
contrast, CAI can be developed in the context of the Kripkean view in an original form (CCAI), which squares with other doctrines of contingent identity.

\section{Acknowledgements}

Some parts of this work have been presented in Milan at the conference of the Società Italiana di Filosofia del Linguaggio in January 2018. We would like to thank the audience, and in particular Lorenzo Azzano, Andrea Borghini and Francesco Orilia, for their questions. We also thank Andrea Borghini, Claudio Calosi, Simone Gozzano, Jeroen Smid and two anonymous reviewers for their insightful comments on earlier versions of the paper.

\section{References}

Barcan, R. (1947). Identity of Individuals in a Strict Functional Calculus of Second Order. Journal of Symbolic Logic, 12(1), 12-15.

Baxter, D. (1988a). Many-One Identity. Philosophical Papers, 17, 193-216.

Baxter, D. (1988b). Identity in the Loose and Popular Sense. Mind, 97, 575-582.

Baxter, D. (2014). Identity, Discernibility, and Composition. In A. Cotnoir \& D. Baxter (Eds.), Composition as Identity (pp. 244-254). Oxford: Oxford University Press.

Bøhn, E. (2009). Composition as Identity: A Study in Ontology and Philosophical Logic. PhD Thesis, University of Massachusetts, Amherst.

Bøhn, E. (2014). Unrestricted Composition as Identity. In A. Cotnoir \& D. Baxter (Eds.), Composition as Identity (pp. 143-165). Oxford: Oxford University Press.

Borghini, A. (2005). Counterpart Theory Vindicated: A Reply to Merricks. Dialectica, 59(1), $67-73$.

Bressan, A. (1972). A General Interpreted Modal Calculus. New Haven: Yale University Press.

Carrara, M. \& Lando, G. (2016). Composition, Indiscernibility, Coreferentiality. Erkenntnis, 81(1), 119-142.

Carrara, M. \& Lando, G. (2017). Composition and Relative Counting. Dialectica, 71(4), 489_ 529.

Carrara, M. \& Martino, E. (2011). Four Theses on the Alleged Innocence of Mereology. Humana.Mente, 4(19), 57-77.

Chisholm, R. (1976). Person and Object: A Metaphysical Study. La Salle, Illinois: Open 
Court Publishing Co.

Cotnoir, A. (2013). Composition as General Identity. In K. Bennett \& D. Zimmerman, Oxford Studies in Metaphysics, Volume 8 (pp. 295-322). Oxford: Oxford University Press.

Cotnoir, A. (2014). Composition as Identity: Framing the Debate. In A. Cotnoir \& D. Baxter (Eds.), Composition as Identity (pp. 3-23). Oxford: Oxford University Press.

Fine, K. (1994). Essence and Modality. Philosophical Perspectives, 8, 1-16.

Gallois, A. (1990). Occasional Identity. Philosophical Studies, 58(3), 203-224.

Gallois, A. (1998). Occasions of Identity. Oxford: Clarendon Press.

Gibbard, A. (1975). Contingent Identity. Journal of Philosophical Logic, 4(2), 187-221.

Graham, A. (2015). From Four to Five-Dimensionalism. Ratio, XXVIII(1), 14-28.

Hewitt, S. T. (2012). Modalising Plurals. Journal of Philosophical Logic, 41(5), 853-875.

Hovda, P. (2005). Two Defenses of Composition as Identity, Unpublished Manuscript. https://www.reed.edu/philosophy/hovda/publications/TwoDefenses.pdf

Kripke, S. (1971). Identity and Necessity. In M. Munitz (Ed.), Identity and Individuation (pp. 135-164). New York: New York University Press.

Kripke, S. (1980). Naming and Necessity. Cambridge (Mass.): Harvard University Press.

Lando, G. (2017). Mereology. A Philosophical Introduction, London: Bloomsbury.

Lewis, D. (1991). Parts of Classes. Oxford: Blackwell.

Linnebo, Ø. (2016). Plurals and Modals. Canadian Journal of Philosophy, 46(4-5), 654-676.

Merricks, T. (1999). Composition as Identity, Mereological Essentialism, and Counterpart Theory. Australasian Journal of Philosophy, 77(2), 192-195.

Merricks, T. (2003). The End of Counterpart Theory. The Journal of Philosophy, 100(10), $521-549$.

Oliver, A., \& Smiley, T. (2013). Plural Logic. Oxford: Oxford University Press.

Plantinga, A. (1974). The Nature of Necessity. Oxford: Oxford University Press.

Quine, W. V. O. (1954). Reference and Modality. Journal of Symbolic Logic, 19(1), 137-138.

Schlesinger, G. (1985). Spatial, Temporal and Cosmic Parts. The Southern Journal of Philosophy, 23(2), 255-271. 
Sider, T. (2007). Parthood. The Philosophical Review, 116(1), 51-91.

Sider, T. (2014). Consequences of Collapse. In A. Cotnoir \& D. Baxter (Eds.), Composition as Identity (pp. 211-221). Oxford: Oxford University Press.

Stalnaker, R. (2003). Ways a World Might Be. Oxford: Oxford University Press.

Torrengo, G. (2011). The Modal Dimension. Humana.Mente, 4(19), 105-120.

Turner, J. (2014). Donald Baxter's Composition as Identity. In A. Cotnoir \& D. Baxter (Eds.), Composition as Identity (pp. 225-243). Oxford: Oxford University Press.

Uzquiano, G. (2011). Plural Quantification and Modality. Proceedings of the Aristotelian Society, 111, 219-250.

Varzi, A. (2001). Parts, Counterparts, and Modal Occurrents. Travaux de logique, 14(1), $151-171$.

Varzi, A. (2008). The Extensionality of Parthood and Composition. The Philosophical Quarterly, 58(230), 108-133.

Wallace, M. (2009). Composition as Identity. PhD Thesis, University of North Carolina, Chapel Hill.

Wallace, M. (2011a). Composition as Identity: Part 1. Philosophy Compass, 6, 804-816.

Wallace, M. (2011b). Composition as Identity: Part 2. Philosophy Compass, 6, 817-827.

Wallace, M. (2014). Composition as Identity, Modal Parts, and Mereological Essentialism. In A. Cotnoir \& D. Baxter (Eds.), Composition as Identity (pp. 111-129). Oxford: Oxford University Press.

Williamson, T. (2010). Necessitism, Contingentism, and Plural Quantification. Mind, 119(475), 657-748.

Williamson, T. (2013). Modal Logic as Metaphysics. Oxford: Oxford University Press.

Yi, B.-U. (1999). Is Mereology Ontologically Innocent? Philosophical Studies, 93(2), 141160. 